\title{
Air travel and incidence of pneumothorax in lymphangioleiomyomatosis
}

\author{
Cynthia Gonano ${ }^{1 \dagger}$, Jérôme Pasquier ${ }^{2 \dagger}$, Cécile Daccord ${ }^{3}$, Simon R. Johnson ${ }^{4}$, Sergio Harari ${ }^{5}$, Violette Leclerc ${ }^{6}$, \\ Lucy Falconer ${ }^{7}$, Eleonora Miano ${ }^{8}$, Jean-François Cordier ${ }^{9}$, Vincent Cottin ${ }^{9}$ (D) and Romain Lazor ${ }^{3^{*}}$ (D)
}

\begin{abstract}
Background: Pulmonary lymphangioleiomyomatosis (LAM) is a rare disease of women characterized by multiple lung cysts leading to respiratory insufficiency and frequent pneumothorax (PT). Air travel (AT) could increase the risk of PT in LAM through rupture of subpleural cysts induced by atmospheric pressure changes in aircraft cabin. To determine whether AT increases the risk of PT in LAM, we performed a retrospective survey of members of European LAM patient associations. A flight-related PT was defined as occurring $\leq 30$ days after AT.

Results: 145 women reported 207 PT. In 128 patients with available data, the annual incidence of PT was 8\% since the first symptoms of LAM and $5 \%$ since LAM diagnosis, compared to $0.006 \%$ in the general female population. Following surgical or chemical pleurodesis, the probability of remaining free of PT recurrence was respectively 82,68 , and $59 \%$ after 1, 5 and 10 years, as compared to only 55,46 and $39 \%$ without pleurodesis $(p=0.026) .70$ patients with available data performed 178 AT. 6 flight-related PT occurred in 5 patients. PT incidence since first symptoms of LAM was significantly higher $\leq 30$ days after AT as compared to non-flight periods (22 versus $6 \%$, risk ratio 3.58 , confidence interval 1.40-7.45).
\end{abstract}

Conclusions: The incidence of PT in LAM is about 1000 times higher than in the general female population, and is further increased threefold after AT. Chemical or surgical pleurodesis partly reduces the risk of PT recurrence in LAM.

Keywords: Lymphangioleiomyomatosis, Incidence, Pneumothorax, Pleurodesis, Air travel

\section{Background}

Pulmonary lymphangioleiomyomatosis (LAM) is a rare disease affecting almost exclusively women in their reproductive age. It is characterised by the proliferation of abnormal smooth muscle-like cells (LAM cells) in the lungs and lymphatic system, and is considered as a low-grade metastasizing neoplasm [1-5]. In the lungs, LAM cell proliferation leads to the development of multiple thin-walled cysts and progressive destruction of the parenchyma resulting in dyspnea, obstructive ventilatory defect, reduced carbon monoxide transfer factor, and hypoxemia [6-10]. Another typical feature of pulmonary LAM is the occurrence of pneumothorax (PT), which affects more than half of patients during disease course with frequent relapses [6-9].

\footnotetext{
* Correspondence: romain.lazor@chuv.ch

${ }^{+}$Cynthia Gonano and Jérôme Pasquier contributed equally to this work

${ }^{3}$ Service de pneumologie, Centre hospitalier universitaire vaudois, PMU

BU44.07, Rue du Bugnon 44, 1011 Lausanne, Switzerland

Full list of author information is available at the end of the article
}

Air travel (AT) is a matter of concern in patients with LAM [11]. During commercial flights, the cruising altitude varies between 4'534 and $14.630 \mathrm{~m}$, and aircraft cabin is pressurized to approximately $565 \mathrm{mmHg}$ corresponding to an altitude of $2438 \mathrm{~m}$. The resulting decrease in alveolar oxygen partial pressure may worsen pre-existing hypoxemia in patients with respiratory diseases. In addition, following Boyle's law, when barometric pressure decreases during ascent, the air eventually trapped in a non-communicating space such as a pulmonary cyst may increase its initial volume by $30 \%$, and could lead to overinflation and rupture, with consecutive PT [12-14]. The occurrence of a PT during flight may have serious consequences in patients with impaired lung function, and its treatment in such circumstances may be delayed. In 2 retrospective studies addressing the issue of AT in patients with LAM, the reported frequency of PT after AT was respectively 2.2 and $1.1 \%$ per flight $[11,15]$. However, these studies did not determine

(c) The Author(s). 2018 Open Access This article is distributed under the terms of the Creative Commons Attribution 4.0 International License (http://creativecommons.org/licenses/by/4.0/), which permits unrestricted use, distribution, and 
whether AT by itself constitutes a risk factor for the occurrence of PT.

To explore this issue, we performed a survey of European LAM patients. The main objectives were to calculate the annual incidence rate of PT in LAM, and to determine whether AT increases the risk of PT in this population.

\section{Methods}

\section{Patient recruitment}

Patients with pulmonary LAM were recruited through European LAM patient associations in France, Germany, Italy, Spain, United Kingdom, and a rare lung disease registry in Switzerland. Isolated patients from other countries were also recruited on an individual basis. Data were collected retrospectively through a questionnaire available in the local language. Patients provided informed consent.

\section{Data collection}

Patients were asked to provide detailed information regarding the date of first symptoms attributable to LAM, date of LAM diagnosis, current forced expiratory volume in one second (FEV1) if known, and the occurrence and date of lung transplantation. They were also asked to report details on each episode of PT, which occurred since the first symptoms of LAM, including date of PT, affected side, and treatment received according to pre-specified categories (spontaneous resolution, needle aspiration, chest tube, or chemical or surgical pleurodesis). Patients were also asked to report with the best possible accuracy the first 4 AT which took place since the first symptoms of LAM, including date, origin and destination, occurrence of PT during or after AT, and whether it occurred on a lung previously treated for PT. Patients were encouraged to provide dates with the best possible accuracy from personal archives (diaries, travel invoices), and obtain details on PT dates and treatments from their physician. The French patient association France Lymphangioléiomyomatose (FLAM) performed data collection and capture. An anonymized database was provided to the investigators. Patients who were identified by the survey as experiencing a $\mathrm{PT} \leq 30$ days after AT received a second questionnaire to ascertain that PT was diagnosed by a physician and by chest $\mathrm{X}$-ray, the dates of $\mathrm{AT}$ and $\mathrm{PT}$, and the treatments received.

\section{Data analysis}

In a first set of analyses, we determined the overall incidence of PT in the study population. The beginning of exposure to the risk of PT was defined as the date of the first symptoms attributable to LAM, and in a second analysis as the date of LAM diagnosis. The end of exposure to the risk of PT was defined as the date of survey completion. When a PT was the first symptom attributable to LAM, it was included in the calculation of PT incidence during the exposure period, which started with the first symptom. We considered 2 different hypotheses to compute PT incidence: 1 ) the risk of PT is constant across the whole LAM population, 2) the risk of PT is variable from one patient to another. A standard Poisson regression (model 1) was used to compute the incidence according to the first hypothesis. To compute PT incidence according to the second hypothesis, we used 2 different regression models to estimate the variable risk [16]: a negative binomial regression (model 2), and a Poisson regression with a random intercept (model 3). In each of these models, only an intercept was considered (mean model). Model 1 is equivalent to calculate the ratio of the total number of observed PT and the sum of all exposure periods. In model 2, we hypothesized that the incidence was distributed as a gamma distribution (the negative binomial distribution can be viewed as a Poisson distribution where the parameter is itself a random variable distributed as a gamma distribution). In this model, the estimation of the intercept leads to an estimation of the mean incidence rate (over the patients). In model 3, we supposed that the intercept was normally distributed and therefore the incidence followed a log-normal distribution. In this model, the estimation of the intercept leads to an estimation of the median incidence rate. Patients were withdrawn from the analysis if the date of the first symptoms, the date of LAM diagnosis, or any date of PT were missing. Transplanted lungs were not considered at higher risk for PT and were withdraw from the calculation.

In a second set of analyses, we determined whether pleurodesis reduced the risk of PT in the study population. For this purpose, we compared the recurrence rate of PT after conservative treatment (spontaneous resolution, needle aspiration or chest tube) and after medical or surgical pleurodesis in patients who experienced a first episode of PT, using the Kaplan-Meier method. Each lung was considered as an independent observation. Patients were withdrawn from the analysis if any date of PT or the affected side were missing, or if they never experienced a PT.

In a third set of analyses, we determined whether AT increased the risk of PT occurrence in LAM. A PT was arbitrarily defined as related to AT if it was diagnosed by chest X-ray within 30 days after AT. This time interval was defined before the survey. Although cyst rupture related to barometric pressure change is expected to occur during AT, the resulting PT (i.e. the leak of a significant amount of air from the airspaces to the pleural cavity through the ruptured cyst) may be delayed, as suggested for another cystic lung disease, the Birth-Hogg-Dubé 
syndrome (BHD) [17]. An interval of up to 30 days has also been observed in a study on the occurrence of PT due to AT in BHD [18]. We also considered that a patient with mild respiratory symptoms may have seeked medical attention only after several days.

Each lung was considered as an independent observation. Patients were withdrawn from the analyses if any date of PT, the affected side, or any date of AT were missing. Based on our (see below) and previous findings [19] that pleurodesis was only moderately effective in reducing the risk of $\mathrm{PT}$ recurrence, lungs treated with pleurodesis were still considered at risk of PT.

An AT was defined as both an outbound and a return trip, each of which may have consisted of one or more stops, i.e. one or more episodes of ascent and descent. The time interval between outbound and return trips was not recorded, but we reasoned that in the vast majority of AT, the duration of a trip would be $<15$ days. Indeed, according to European statistics, the average duration of trips performed by European citizen is 5.4 days [20], and $94 \%$ of trips last $<14$ days [21]. Thus, the interval of 30 days after the outbound trip was considered appropriate to observe the occurrence of PT related to an AT. The date of AT, defined as the date of the first outbound flight, was considered as day 0 .

To determine whether AT increased the risk of PT occurrence, we compared the incidence of PT during the 30 days following AT (days 0 to +29 ) to the incidence of PT at all other periods, i.e. before AT (from first symptoms or diagnosis) and $>30$ days after AT. A standard Poisson regression was used for these analyses. Quantitative data were expressed as mean and standard deviation (SD). Statistical analyses were performed with the $\mathrm{R}$ software version 3.4.4 [22].

\section{Results}

\section{Study population}

145 filled questionnaires were available. All patients were women. Their countries of origin were France (31.7\%), Germany (23.4\%), United Kingdom (17.2\%), Italy (15.2\%), Spain (5.5\%), Switzerland (4.1\%), Austria (0.7\%), Belgium (0.7\%), Ireland (0.7\%), and Turkey (0.7\%). The response rate, available for the French association, was $51 \%$. The mean (SD) age at time of survey was 47 (12) years. The mean (SD) age was 36 (11) years at first symptoms attributable to LAM $(n=139)$, and 41
(11) years at LAM diagnosis $(n=145)$. The mean (SD) $\mathrm{FEV}_{1}$ at time of survey was $58(24) \%$ predicted $(n=71)$.

\section{Incidence of pneumothorax in LAM}

Among the 145 patients, 6 had missing dates of first symptoms of LAM, and 11 had one or more dates of PT missing. In the remaining 128 patients, the mean follow-up duration since the first symptoms attributable to LAM was 11.4 years, and the cumulated follow-up duration was 1454 patient-years. The mean follow-up duration since LAM diagnosis was 6.4 years, with a cumulated follow-up duration of 817 patient-years.

The 145 patients reported a total of 207 PT. Eighty-three patients (57\%) had at least one PT, and 56 (39\%) had 2 or more PT. Among the 137 patients for whom the side of each PT was determined, the mean (SD) number of PT per lung was 1.8 (1.0).

In the 128 patients with available data, the annual incidence rate of PT since the first symptoms of LAM and since LAM diagnosis according to the 3 statistical models are shown in Table 1. Since the number of PT varied widely among patients, we considered that model 3 was the most appropriate to describe the incidence of PT. With this model, the annual incidence of PT was $8 \%$ since first symptoms of LAM, and 5\% since LAM diagnosis. Higher rates were found with the 2 other statistical models (Table 1), showing that model 3 was the most conservative.

\section{Efficacy of pleurodesis to prevent recurrence of PT}

The probability of remaining free of PT recurrence without pleurodesis was $55 \%$ at one year, $46 \%$ at 5 years and $39 \%$ at 10 years after the initial PT, versus 82,68 and $59 \%$ with pleurodesis, respectively $(p=0.026$, Log rank, Kaplan-Meier method) (Fig. 1). No significant difference was observed between chemical and surgical pleurodesis $(p=0.69$, data not shown). As the protective effect of pleurodesis was only partial, and for the purpose of calculating the risk of PT after AT, we considered that a lung treated with pleurodesis remained at risk of PT afterwards.

\section{Risk of pneumothorax after air travel}

Eighty-three out of 145 patients (57\%) travelled by air. Among them, 3 (cases 26, 36, and 79) had missing or incomplete dates of AT, 3 (cases 38, 53, and 89) had

Table 1 Annual incidence rate of pneumothorax in LAM $(n=128)$

\begin{tabular}{llll}
\hline Start of exposure period & Model 1 & Model 2 & Model 3 \\
\hline First symptoms of LAM & $0.12(0.1,0.14)$ & $0.18(0.13,0.24)$ & $0.08(0.05,0.11)$ \\
LAM diagnosis & $0.09(0.07,0.11)$ & $0.11(0.07,0.15)$ & $0.05(0.03,0.08)$ \\
\hline
\end{tabular}

Data were calculated from 3 models: Model 1: constant risk of PT across the whole LAM population, standard Poisson regression. Model 2: variable risk from one patient to another, negative binomial regression. Model 3: variable risk from one patient to another, Poisson regression with random intercept. Results are expressed as incidence per patient per year (95\% confidence intervals) 


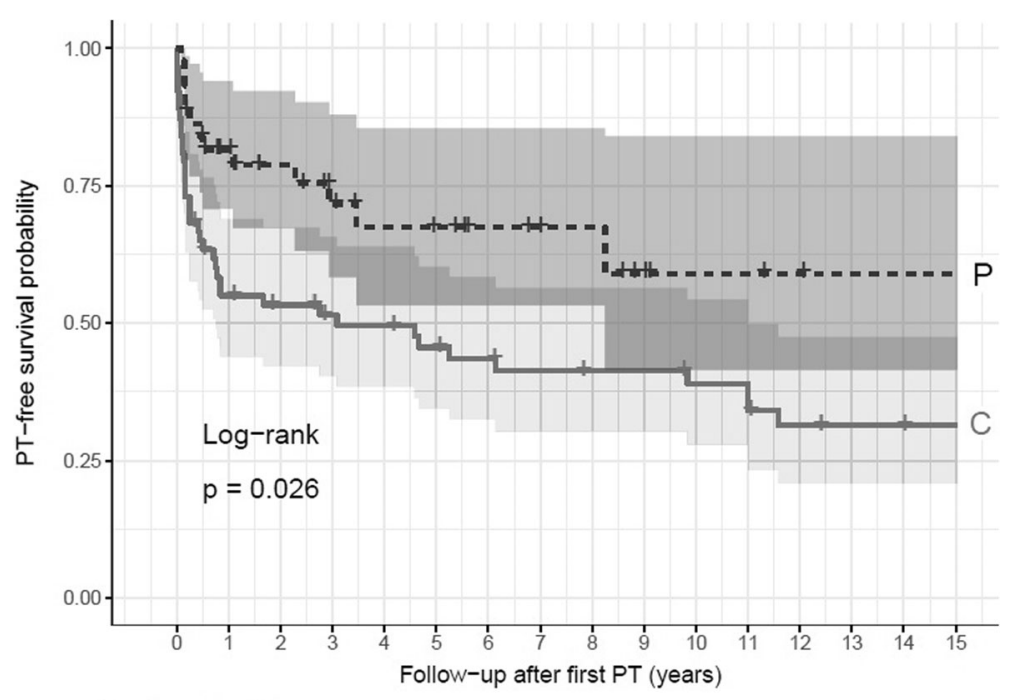

Number at risk

$$
\begin{array}{lllllllllllllllll}
\mathrm{C} & 63 & 33 & 30 & 27 & 26 & 23 & 21 & 19 & 18 & 18 & 16 & 15 & 12 & 11 & 11 & 9 \\
\mathrm{P} & 44 & 30 & 24 & 19 & 16 & 14 & 10 & 9 & 8 & 5 & 3 & 3 & 2 & 1 & 1 & 1
\end{array}
$$

Fig. 1 Probability of pneumothorax-free course after the first pneumothorax, according to treatment received for this pneumothorax. Each lung was considered as a separate observation. Probability of pneumothorax-free course was significantly lower after conservative treatment ( $C$, grey solid line) than after chemical or surgical pleurodesis ( $P$, black broken line) ( $p=0.026$, log-rank test). Grey areas reflect 5-95\% confidence intervals. Data were censored at the date of questionnaire filling if a second pneumothorax did not occur. PT: pneumothorax

missing or incomplete dates of PT, and 4 (cases 11, 38, 89, and 93) had missing side of PT. In one other patient (case 141), a PT was reported on the same day as an AT, but this event was not counted since all AT $(n=3)$ and PT $(n=4)$ in this patient were reported as occurring on January 1st, which was considered very unlikely. Moreover, 4 patients had missing dates of first symptoms of LAM or did not fly after first symptoms of LAM (cases $31,75,97,144)$. Thus, after removing these 13 cases, a population of 70 patients was used to determine the risk of PT associated with AT after the first symptoms of LAM. These 70 patients reported a total of 178 AT. Considering each lung as an independent observation, 140 lungs performed a total of 356 AT. After LAM diagnosis, 57 patients performed 139 AT, or 114 lungs performed 278 AT.

Six PT occurred in 5 patients (bilateral PT in one patient revealing the disease) within 30 days after AT (Table 2), including 4 patients who experienced their first PT at this occasion, and one who had 3 PT previously and relapsed on the same side despite previous chemical pleurodesis. Three other patients (cases 27, 98, and 107) mentioned the occurrence of PT after AT in comments, but since this was not consistent with the reported dates of PT and AT, these narratively mentioned PT were not counted. Overall, the rate of PT within 30 days after AT was $2.8 \%(5 / 178)$ per patient and $1.7 \%(6 / 356)$ per lung after first symptoms of LAM. After LAM diagnosis, the rate of
PT within 30 days after AT was 2.2\% (3/139) per patient and $1.1 \%(3 / 278)$ per lung.

The incidence of PT within 30 days after AT was compared to the incidence of PT during all non-flight periods since first symptoms of LAM, and since LAM diagnosis, respectively (Table 3 ). When using the first symptoms of LAM as the beginning of the period at risk, a significantly higher incidence of PT was found within 30 days after AT $(0.223$, CI $0.089-0.453)$ as compared to non-flight periods $(0.062$, CI $0.052-0.075)$ with a risk ratio of 3.58 (CI 1.40-7.45). When using the date of LAM diagnosis as the beginning of the period at risk, the incidence of PT was also increased within 30 days after AT (0.143, CI 0.035-0.370) as compared to non-flight periods $(0.044$, CI 0.032-0.059), but the difference did not reach statistical significance (risk ratio 3.25, CI 0.798.93). No PT was counted twice because of overlapping periods at risk.

\section{Discussion}

In the present study, we determined for the first time the incidence of PT in LAM, which is about 1000 times higher than in the general women population. Another new finding is that the incidence of PT is increased threefold after AT compared to baseline incidence, suggesting that AT could be a risk factor for the occurrence of PT in LAM. Additionally, we confirm previous data showing that pleurodesis is partly effective in reducing the recurrence rate of PT in LAM [19]. 
Table 2 Characteristics of LAM patients who presented PT within 30 days after AT

\begin{tabular}{|c|c|c|c|c|c|c|}
\hline $\begin{array}{l}\text { Case } \\
\text { number }\end{array}$ & $\begin{array}{l}\text { Age at PT } \\
\text { (years) }\end{array}$ & $\begin{array}{l}\text { Number of previous } \\
\text { PT }\end{array}$ & $\begin{array}{l}\text { Side of current } \\
\text { PT }\end{array}$ & $\begin{array}{l}\text { Delay between AT and PT } \\
\text { (days) }\end{array}$ & $\begin{array}{l}\text { Perceived relationship } \\
\text { between AT and PT }\end{array}$ & PT treatment \\
\hline 143 & 26 & 0 & $R$ & 0 & yes & surgical pleurodesis \\
\hline 143 & 26 & 0 & L & 0 & yes & surgical pleurodesis \\
\hline 84 & 64 & 0 & $\mathrm{R}$ & 19 & yes & $\begin{array}{l}\text { chemical } \\
\text { pleurodesis }\end{array}$ \\
\hline 25 & 26 & 0 & L & 24 & yes & surgical pleurodesis \\
\hline 102 & 44 & 3 & $\mathrm{R}$ & 29 & no & $\begin{array}{l}\text { spontaneous } \\
\text { healing }\end{array}$ \\
\hline 142 & 59 & 0 & $\mathrm{R}$ & 29 & yes & surgical pleurodesis \\
\hline
\end{tabular}

PT Pneumothorax, AT air travel, $L$ Left, $R$ Right

Several series have reported that 50 to $80 \%$ of LAM patients experience PT during disease course [6-9], but the incidence of PT in LAM has not been determined previously. Based on the wide interindividual variation in the number of PT observed in the present study (range $0-4$ ), we considered that the risk of PT would be variable from one patient to another, and as the median is more robust to outliers than the mean, model 3 was considered the most appropriate. With this model, the incidence of PT in the LAM population was $8 \%$ per year from the first symptoms of LAM, and 5\% per year from LAM diagnosis. In comparison, the incidence of spontaneous PT in the general female population is 1 to $6 / 100^{\prime} 000$ per year, or 0.001 to $0.006 \%$ [23]. Using the most conservative estimate, the incidence of PT in LAM is therefore about 1000 times higher than in the general population.

Only one retrospective survey has previously examined the efficacy of pleurodesis to prevent PT recurrence in LAM [19]. Among 301 episodes of first PT in 193 patients, the recurrence rate was $66 \%$ after conservative therapy, 27\% after chemical pleurodesis and 32\% after surgical pleurodesis [19]. In the present study, we confirm that pleurodesis significantly reduces the risk of PT recurrence in LAM (Fig. 1), and that chemical and surgical pleurodesis are of similar efficacy. However, the risk of recurrence after pleurodesis remained much higher than in spontaneous primary $\mathrm{PT}$, with reported recurrence rates of 0 to $3.2 \%$ after surgical pleurodesis, and
2.5 to $10 \%$ after thoracoscopic talc poudrage [24]. Based on these findings, we considered that LAM patients with pleurodesis remain at risk of $\mathrm{PT}$ after $\mathrm{AT}$, and did not exclude post-pleurodesis periods from calculations. Indeed, among the 5 patients who had a PT within 30 days after AT, one had a previous pleurodesis on the same side.

The incidence of PT during AT aboard commercial aircrafts in the general population is unknown but probably very low. Only $0.003 \%$ of passengers have an in-flight medical problem requiring emergency intervention [25], and PT is usually not mentioned in studies on in-flight emergencies [25-27]. However, there are several case reports of in-flight PT [14, 25, 28-30]. Furthermore, a relationship between atmospheric pressure changes and occurrence of spontaneous PT has been demonstrated in several studies [31-34]. A case of spontaneous PT triggered by an ascent of $350 \mathrm{~m}$ in a high-speed lift has also been reported [35]. In the US Air Force personnel, the incidence of spontaneous PT was $47 / 100^{\prime} 000 /$ year [36], i.e. fourfold higher than in the US Navy [36] or the general male population [23]. Taken together, these data suggest that variations of atmospheric pressure during AT may trigger PT. Although the risk appears very low in the general population, it may be higher in subjects prone to PT, such as LAM patients. Lung function testing has also been shown to trigger PT in LAM patients with an incidence rate of 0.02 to $0.04 /$ 100 tests [37], which is certainly much higher than in

Table 3 Incidence of PT during post-flight and non-flight periods

\begin{tabular}{lllll}
\hline $\begin{array}{l}\text { Start of exposure } \\
\text { period }\end{array}$ & $\begin{array}{l}\text { Exposure period } \\
\text { type }\end{array}$ & $\begin{array}{l}\text { Exposure period duration } \\
\text { (lung-yr) }\end{array}$ & $\begin{array}{l}\text { Number of PT during } \\
\text { exposure period }\end{array}$ & $\begin{array}{l}\text { PT incidence per lung } \\
\text { per yr (Cl) }\end{array}$ \\
\hline first symptoms of LAM & total & 1835.4 & 119 & $0.065(0.054-0.077)$ \\
& non-flight & 1808.6 & 113 & $0.062(0.052-0.075)$ \\
& 30 days post-flight & 26.8 & 6 & $0.223(0.089-0.453)$ \\
LAM diagnosis & total & 930.7 & 43 & $0.046(0.034-0.061)$ \\
& non-flight & 909.7 & 40 & $0.044(0.032-0.059)$ \\
& 30 days post-flight & 21.0 & 3 & $0.143(0.035-0.370)$ \\
\hline
\end{tabular}


the general population. To our knowledge, despite the extremely wide use of this procedure, only 3 cases of PT after lung function testing have been reported in the literature [38-40].

Two previous studies have analysed the occurrence of PT related to AT in LAM patients [11, 15]. The first study surveyed members of the LAM Foundation (USA) and the LAM Action registry (UK) who travelled by air. The rate of PT was estimated to $4 \%$ per patient and $2.2 \%$ per flight [11]. In another study of LAM patients who travelled to the National Institutes of Health, the rate of PT related to AT was $2.9 \%$ per patient and $1.1 \%$ per flight, compared to $1.3 \%$ per patient and $0.5 \%$ per journey with ground travel [15]. The rate of PT in the present study $(2.8 \%$ per patient per flight and $1.7 \%$ per lung per flight) is consistent with these previous findings $[11,15]$, thus reinforcing the validity of our observations. However, these previous studies did not provide any information on a possible causal relationship between AT and PT because no comparison was made between patients who flew and those who did not fly $[11,15]$.

In contrast, and for the first time, we compared the incidence of PT after AT with the baseline incidence of PT in LAM, to assess the risk of PT attributable to AT. We observed a significant threefold increase of PT incidence $\leq 30$ days after AT as compared to non-flight periods (22 versus $6 \%$ per year, risk ratio 3.58 , CI 1.40-7.45) when using the date of first symptoms as the beginning of the period at risk. A similar threefold increase was observed when the period at risk was calculated from the date of diagnosis (14 versus $4 \%$ per year, risk ratio 3.25 , CI $0.79-8.93)$, although the difference did not reach statistical significance due to a smaller sample size. We however believe that the date of first symptoms is more relevant than the date of diagnosis to define the period a risk in LAM, since PT is the first disease manifestation in about half of cases, and diagnosis is often delayed by several years [6, 7]. Indeed, in the present study, the mean age at first symptoms was 36 years, whereas the mean age at diagnosis was 41 years. Discarding the period between first symptoms and diagnosis would have led to miss a time span during which patients are already exposed to the risk of PT, as well as a significant proportion of events. Indeed, among the 5 patients who experienced PT after AT, one had bilateral PT after AT as the first disease manifestation. Altogether, our findings suggest for the first time that AT by itself could be a risk factor for PT occurrence in LAM.

The 2010 European Respiratory Society guidelines on LAM provided recommendations regarding AT [41]. LAM patients with minimal respiratory manifestations were not discouraged to fly, unless they presented new respiratory symptoms not evaluated by a physician. Patients with a known untreated PT or a PT treated within the previous month were advised not to travel by air. We believe that our findings should not lead to modify these recommendations, as the additional risk of AT is much smaller than the baseline risk of PT in LAM. However, patients should be informed of this additional risk.

Recently, Johannesma et al. evaluated by questionnaires the risk of spontaneous PT due to AT in patients with BHD, an autosomal genetic disease characterised by skin lesions, renal tumors and multiple pulmonary cysts $[11,15,18]$. From the 145 patients who flew, 13 presented a PT confirmed by chest X-ray $\leq 1$ month after AT. The risk was $0.63 \%$ per flight, i.e. lower than in LAM (11, 15, 18, and the present study). Consistently, the frequency of PT during disease course is lower in BHD (35-38\%) than in LAM (50-80\%) [6-9, 19, 37, 42, 43], a difference possibly explained by less numerous cysts in BHD, or cysts less prone to rupture [18].

The present study has several strengths. We studied a relatively large cohort of patients with LAM from various countries. We considered each lung as an independent observation, thus allowing to analyse particular situations such as bilateral PT. We restricted the analyses to cases with available dates of events and excluded those with missing, incomplete or doubtful dates, even if a PT was narratively reported as occurring after AT $(n=3)$. By using a 30-days interval as the period at risk for PT occurrence after $\mathrm{AT}$, we took into account the fact that PT occurrence and diagnosis may be delayed, as previously reported [17].

Our study has several limitations. As the study design was a patient survey, we did not check the accuracy of the diagnosis. However, since patients were members of LAM associations, we assumed that LAM was the correct diagnosis in all. As study participants were lay persons, one could argue that they did not have enough knowledge to accurately fill the questionnaire. However, participants were not a sample of the general population, but young adults affected by a rare disease, and members of LAM patient associations, which provide regular educational sessions on LAM to their members. These patients were therefore well informed of PT mechanisms, symptoms, diagnosis and therapy. We recorded a maximum of $4 \mathrm{AT}$ and $4 \mathrm{PT}$ episodes, which may have led to underestimate the number of events. However, the number of patients who experienced $4 \mathrm{PT}$ on the same side was small $(n=10)$, and we believe that we did not miss an important proportion of events. Recall bias and errors in questionnaire filling might have occurred. However, we checked by a second questionnaire the validity of the data regarding PT occurring after AT. 
Patients who replied to the survey may not be fully representative of the whole LAM population. Furthermore, patients who experienced symptoms during AT or feared the occurrence of PT may have been more prone to respond to the survey. The number of events was small, and a larger sample would be required to measure more accurately the risk of PT occurrence after AT. We acknowledge that, even if used in one previous study on PT and AT in BHD [18], the 30-days interval used to define the period at risk of PT after AT was arbitrary. However, shorter intervals would have led to a reduced number of events and an underpowered analysis, whereas longer intervals would have obfuscated AT-related PT among spontaneous PT. Finally, other causes of decreased barometric pressure, such as meteorological changes or ascent to high altitude, were not accounted. Despite these methodological limitations, we believe that our approach provides a valuable new insight in a poorly studied phenomenon.

\section{Conclusion}

The annual incidence of PT in the LAM population was $8 \%$ per year since the first symptoms and $5 \%$ per year since LAM diagnosis, i.e. around 1000 times higher than the risk of spontaneous PT in the general female population. Pleurodesis after the first PT partially but significantly reduced the risk of subsequent PT. The probability of PT within 30 days after AT was increased threefold as compared to periods without AT, suggesting for the first time that AT by itself could be a risk factor for PT occurrence in LAM. This study also illustrates the valuable role of patient associations in research on rare diseases.

\section{Abbreviations}

AT: air travel; BHD: Birt-Hogg-Dubé syndrome; Cl: confidence interval; FEV1: forced expiratory volume in one second; FLAM: France Lymphangioléiomyomatose (French patient association):

LAM: Iymphangioleiomyomatosis; PT: pneumothorax; SD: standard deviation

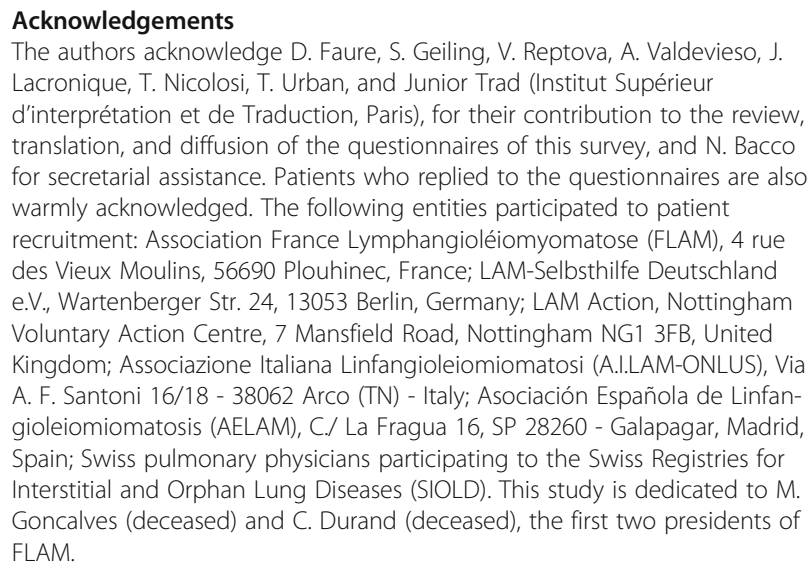
translation, and diffusion of the questionnaires of this survey, and N. Bacco for secretarial assistance. Patients who replied to the questionnaires are also warmly acknowledged. The following entities participated to patient recruitment: Association France Lymphangioléiomyomatose (FLAM), 4 rue des Vieux Moulins, 56690 Plouhinec, France; LAM-Selbsthilfe Deutschland e.V., Wartenberger Str. 24, 13053 Berlin, Germany; LAM Action, Nottingham Voluntary Action Centre, 7 Mansfield Road, Nottingham NG13FB, United Kingdom; Associazione Italiana Linfangioleiomiomatosi (A.I.LAM-ONLUS), Via A. F. Santoni 16/18 - 38062 Arco (TN) - Italy; Asociación Española de Linfangioleiomiomatosis (AELAM), C./ La Fragua 16, SP 28260 - Galapagar, Madrid, Spain; Swiss pulmonary physicians participating to the Swiss Registries for Interstitial and Orphan Lung Diseases (SIOLD). This study is dedicated to M. Goncalves (deceased) and C. Durand (deceased), the first two presidents of FLAM

\section{Funding}

This study was funded with internal resources only.

\section{Availability of data and materials}

The 2 patient questionnaires and the dataset used during the current study are available from the corresponding author on reasonable request.

\section{Authors' contributions}

Study conception and design: JFC, RL, VC; data collection: VL, LF, EM, RL, SJ, $\mathrm{SH}$; data analysis and interpretation: JP, RL, CG, CD, SJ, SH, VC; manuscript drafting: $C G, R L, C D$, JP; critical manuscript revision: all authors; final manuscript approval: all authors.

\section{Ethical approval and consents to participate}

All patients provided written informed consent. As the data collection was initiated by patient associations themselves and took place in several European countries, ethical approval was waived.

\section{Consent for publication}

Not applicable.

\section{Competing interests}

CG has nothing to disclose. JP has nothing to disclose. CD has nothing to disclose. SJ reports personal fees from Novartis, outside the submitted work. $\mathrm{SH}$ reports personal fees from Roche, grants and personal fees from Actelion and Boehringer-Ingelheim, outside the submitted work. VL has nothing to disclose. LF has nothing to disclose. EM has nothing to disclose. JFC has nothing to disclose. VC reports personal fees from Novartis, outside the submitted work. RL reports personal fees from Boehringer-Ingelheim, grants and personal fees from Roche, personal fees from Vifor, outside the submitted work.

\section{Publisher's Note}

Springer Nature remains neutral with regard to jurisdictional claims in published maps and institutional affiliations.

\section{Author details}

'Service de médecine interne, Hôpital neuchâtelois, La Chaux-de-Fonds, Switzerland. Institut de médecine sociale et préventive, Centre hospitalier universitaire vaudois, Lausanne, Switzerland. ${ }^{3}$ Service de pneumologie, Centre hospitalier universitaire vaudois, PMU BU44.07, Rue du Bugnon 44, 1011 Lausanne, Switzerland. ${ }^{4}$ National Centre for

Lymphangioleiomyomatosis, University of Nottingham, Nottingham, United Kingdom. ${ }^{5}$ U.O. di Pneumologia e Terapia Semi-Intensiva Respiratoria,

Servizio di Fisiopatologia Respiratoria ed Emodinamica Polmonare, Ospedale San Giuseppe, MultiMedica IRCCS, Milan, Italy. ${ }^{6}$ Association France Lymphangioléiomyomatose (FLAM), Plouhinec, France. ${ }^{7}$ LAM Action, Nottingham, United Kingdom. ${ }^{8}$ Associazione Italiana Linfangioleiomiomatosi (A.I.LAM-ONLUS), Arco, Italy. ${ }^{9}$ National Reference center for rare pulmonary diseases, Claude Bernard University Lyon 1, OrphaLung, UMR 754, Lyon, France.

Received: 1 October 2018 Accepted: 26 November 2018

Published online: 13 December 2018

\section{References}

1. Gupta N, Vassallo R, Wikenheiser-Brokamp KA, McCormack FX. Diffuse cystic lung disease. Part I. Am J Respir Crit Care Med. 2015;191:1354-66.

2. Taveira-DaSilva AM, Moss J. Clinical features, epidemiology, and therapy of lymphangioleiomyomatosis. Clin Epidemiol. 2015;7:249-57.

3. Johnson SR, Taveira-DaSilva AM, Moss J. Lymphangioleiomyomatosis. Clin Chest Med. 2016:37:389-403.

4. Krymskaya VP, McCormack FX. Lymphangioleiomyomatosis: a monogenic model of malignancy. Annu Rev Med. 2017;68:69-83.

5. Daccord C, Nicod LP, Lazor R. Cystic lung disease in genetic syndromes with deficient tumor suppressor gene function. Respiration. 2017;94:467-85.

6. Taylor JR, Ryu J, Colby TV, Raffin TA. Lymphangioleiomyomatosis. Clinical course in 32 patients. N Engl J Med. 1990;323:1254-60.

7. Urban T, Lazor R, Lacronique J, Murris M, Labrune S, Valeyre D, Cordier JF, The Groupe d'Etudes et de Recherche sur les maladies "Orphelines" Pulmonaires (GERMOP). Pulmonary lymphangioleiomyomatosis. A study of 69 patients. Medicine (Baltimore). 1999;78:321-37.

8. Johnson SR, Tattersfield AE. Clinical experience of Iymphangioleiomyomatosis in the UK. Thorax. 2000;55:1052-7. 
9. Ryu JH, Moss J, Beck GJ, Lee JC, Brown KK, Chapman JT, et al. The NHLBI lymphangioleiomyomatosis registry: characteristics of 230 patients at enrollment. Am J Respir Crit Care Med. 2006;173:105-11.

10. Lazor R, Valeyre D, Lacronique J, Wallaert B, Urban T, Cordier JF, The Groupe d'Etudes et de Recherche sur les maladies "Orphelines" Pulmonaires (GERMOP). Low initial KCO predicts rapid FEV1 decline in pulmonary lymphangioleiomyomatosis. Respir Med. 2004;98:536-41.

11. Pollock-BarZiv S, Cohen MM, Downey GP, Johnson SR, Sullivan E, McCormack FX. Air travel in women with lymphangioleiomyomatosis. Thorax. 2007;62:176-80.

12. Baumann MH. Pneumothorax and air travel: lessons learned from a bag of chips. Chest. 2009;136:655-6.

13. Bunch A, Duchateau FX, Verner L, Truwit J, O'Connor R, Brady W. Commercial air travel after pneumothorax: a review of the literature. Air Med J. 2013;32:268-74

14. Hu X, Cowl CT, Baqir M, Ryu JH. Air travel and pneumothorax. Chest. 2014 145:688-94.

15. Taveira-DaSilva AM, Burstein D, Hathaway OM, Fontana JR, Gochuico BR, Avila NA, et al. Pneumothorax after air travel in lymphangioleiomyomatosis, idiopathic pulmonary fibrosis, and sarcoidosis. Chest. 2009;136:665-70.

16. Thomsen JL, Parner ET. Methods for analysing recurrent events in health care data. Examples from admissions in Ebeltoft health promotion project. Fam Pract. 2006;23:407-13.

17. Postmus PE, Johannesma PC, Menko FH, Paul MA. In-flight pneumothorax: diagnosis may be missed because of symptom delay. Am J Respir Crit Care Med. 2014;190:704-5.

18. Johannesma PC, van de Beek I, van der Wel JW, Paul MA, Houweling AC, Jonker MA, et al. Risk of spontaneous pneumothorax due to air travel and diving in patients with Birt-Hogg-Dube syndrome. Springerplus. 2016;5:1506.

19. Almoosa KF, Ryu JH, Mendez J, Huggins JT, Young LR, Sullivan EJ, et al. Management of pneumothorax in lymphangioleiomyomatosis: effects on recurrence and lung transplantation complications. Chest. 2006;129:1274-81.

20. Eurostat: Products Statistics in Focus: Tourism in Europe: Results for 2011 Issue number 28/2012. Available from: http://ec.europa.eu/eurostat/web/ products-statistics-in-focus/-/KS-SF-12-028

21. Eurostat. Trips made by EU-28 residents by duration, destination and purpose, 2015. Available from: http://ec.europa.eu/eurostat/statisticsexplained/index.php?title=File:Trips_made_by_EU-28_residents_by_ duration,_destination_and_purpose,_2015.png

22. R Core Team. R: A language and environment for statistical computing. $\mathrm{R}$ Foundation for Statistical Computing, Vienna, Austria, 2016. Available from: https://www.R-project.org

23. Gupta D, Hansell A, Nichols T, Duong T, Ayres JG, Strachan D. Epidemiology of pneumothorax in England. Thorax. 2000;55:666-71.

24. Hallifax RJ, Yousuf A, Jones HE, Corcoran JP, Psallidas I, Rahman NM. Effectiveness of chemical pleurodesis in spontaneous pneumothorax recurrence prevention: a systematic review. Thorax. 2016;72:1121-31.

25. Speizer C, Rennie CJ 3rd, Breton H. Prevalence of in-flight medical emergencies on commercial airlines. Ann Emerg Med. 1989;18:26-9.

26. Cummins RO, Schubach JA. Frequency and types of medical emergencies among commercial air travelers. JAMA. 1989;261:1295-9.

27. Peterson DC, Martin-Gill C, Guyette FX, Tobias AZ, McCarthy CE, Harrington ST, et al. Outcomes of medical emergencies on commercial airline flights. N Engl J Med. 2013;368:2075-83.

28. Amdur RD. Recurrent spontaneous pneumothorax caused by aerial flight: report of case. J Aviat Med. 1956;27:456-9.

29. Flux M, Dille JR. Inflight spontaneous pneumothorax: a case report. Aerosp Med. 1969;40:660-2

30. Neidhart P, Suter PM. Pulmonary bulla and sudden death in a young aeroplane passenger. Intensive Care Med. 1985;11:45-7.

31. Bense L. Spontaneous pneumothorax related to falls in atmospheric pressure. Eur J Respir Dis. 1984;65:544-6.

32. Scott GC, Berger R, McKean HE. The role of atmospheric pressure variation in the development of spontaneous pneumothoraces. Am Rev Respir Dis. 1989;139:659-62

33. Bulajich B, Subotich D, Mandarich D, Kljajich RV, Gajich M. Influence of atmospheric pressure, outdoor temperature, and weather phases on the onset of spontaneous pneumothorax. Ann Epidemiol. 2005;15:185-90.

34. Alifano M, Forti Parri SN, Bonfanti B, Arab WA, Passini A, Boaron M, et al. Atmospheric pressure influences the risk of pneumothorax: beware of the storm! Chest. 2007;131:1877-82.
35. Araki $\mathrm{K}$, Okada $\mathrm{Y}$, Kono $\mathrm{Y}$, To $\mathrm{M}$, To $\mathrm{Y}$. Pneumothorax recurrence related to high-speed lift. Am J Med 2014;127:e11-e12.

36. Fuchs HS. Incidence of spontaneous pneumothorax in apparently healthy aircrew. Riv Med Aeronaut Spaz. 1979;42:428-42.

37. Taveira-DaSilva AM, Julien-Williams P, Jones AM, Moss J. Incidence of pneumothorax in patients with lymphangioleiomyomatosis undergoing pulmonary function and exercise testing. Chest. 2016;150:e5-8.

38. Finegan BA, McFarlane HJ. Unusual complication of pulmonary function testing. Anaesthesia. 1983;38:178-9.

39. Manco JC, Terra-Filho J, Silva GA. Pneumomediastinum, pneumothorax and subcutaneous emphysema following the measurement of maximal expiratory pressure in a normal subject. Chest. 1990;98:1530-2.

40. Araujo MS, Fernandes FL, Kay FU, Carvalho CR. Pneumomediastinum, subcutaneous emphysema, and pneumothorax after a pulmonary function testing in a patient with bleomycin-induced interstitial pneumonitis. J Bras Pneumol. 2013;39:613-9.

41. Johnson SR, Cordier JF, Lazor R, Cottin V, Costabel U, Harari S, et al. European Respiratory Society guidelines for the diagnosis and management of lymphangioleiomyomatosis. Eur Respir J. 2010;35:14-26.

42. Toro JR, Wei MH, Glenn GM, Weinreich M, Toure O, Vocke C, et al. BHD mutations, clinical and molecular genetic investigations of Birt-Hogg-Dube syndrome: a new series of 50 families and a review of published reports. J Med Genet. 2008;45:321-31.

43. Gupta N, Seyama K, McCormack FX. Pulmonary manifestations of Birt-HoggDube syndrome. Familial Cancer. 2013;12:387-96.

\section{Ready to submit your research? Choose BMC and benefit from:}

- fast, convenient online submission

- thorough peer review by experienced researchers in your field

- rapid publication on acceptance

- support for research data, including large and complex data types

- gold Open Access which fosters wider collaboration and increased citations

- maximum visibility for your research: over $100 \mathrm{M}$ website views per year

At $\mathrm{BMC}$, research is always in progress.

Learn more biomedcentral.com/submissions 